\title{
Estrategias de generalización de patrones y sus diferentes formas de uso en quinto grado
}

\section{Pattern generalization strategies and their different forms of use in fifth grade}

Melby Cetina-Vázquez, Guadalupe Cabañas-Sánchez

Centro de Investigación en Matemática Educativa, Universidad Autónoma de Guerrero, México

mgcetinav@uagro.mx,gcabanas@uagro.mx

RESUMEN • El artículo reporta las estrategias utilizadas por niños de quinto grado de primaria, junto con las diferentes formas de uso en la resolución de tres problemas de generalización de patrones lineales. El estudio se sustenta en las formas de proceder y en las diferentes formas de representación que usaron para establecer una conclusión o responder a una cuestión. Se identificaron siete tipos de estrategias y quince formas de su uso, examinadas durante seis sesiones en un experimento de enseńanza. Los resultados destacan que las estrategias de contar y recursiva ayudaron a los niños en el uso de estrategias como las de diferencia, objeto-entero, fragmentar, funcional y otras. Estas estrategias presentan de una a tres diferentes formas de uso, lo que manifiesta la diversidad de caminos utilizados por los niños en la generalización de patrones lineales.

PALABRAS CLAVE: Generalización; Patrones lineales; Estrategias; Formas de usos; Experimento de enseñanza.

ABSTRACT • The article reports the strategies used by fifth grade primary school students, along with the different forms of use in solving three linear pattern generalization problems. The study is based on the proceeding forms and the different representational forms used to stablish a conclusion or answer a question. Seven types of strategies and fifteen forms of their use were identified and examined during six sessions in a teaching experiment. The findings highlight that counting and recursive strategies contributed in the children towards the use of strategies such as the difference, whole-object, chunking, functional and others. These strategies present from one to three different forms of use, thus manifesting the variety of paths used by children in the generalization of linear patterns.

KEYWORDS: Generalization; Linear patterns; Strategies; Forms of use; Teaching experiment.

Recepción: septiembre 2019• Aceptación: agosto 2021

Cetina-Vázquez, M. y Cabañas-Sánchez, G. (2022). Estrategias de generalización de patrones y sus diferentes formas de uso en quinto grado. Enseñanza de las Ciencias, 40(1), 65-86. 


\section{INTRODUCCIÓN}

El contexto del estudio es el álgebra temprana, propuesta curricular que sugiere incorporar en las aulas de educación primaria actividades dirigidas al estudio y generalización de patrones, relaciones y propiedades matemáticas que potencien competencias propias del álgebra (Blanton y Kaput, 2005). Su propósito es desarrollar el pensamiento algebraico desde edades tempranas, a fin de que los nińos pequeños experimenten una matemática diferente en la escuela primaria, mediante actividades que les permitan ir más allá del trabajo aislado con números, propiedades y cálculos aritméticos. Y a partir de ello, que incrementen su comprensión sobre la forma estructural y la generalidad de las matemáticas, así como su probabilidad de éxito en el estudio de las matemáticas más avanzadas, especialmente el álgebra, en educación secundaria (Blanton et al., 2015).

El pensamiento algebraico es conceptualizado por Smith (2008) como procesos mentales a través de los cuales un individuo crea un significado referencial para algún tipo de representación, y al hacerlo, construye y expresa generalizaciones. Una ruta para su desarrollo es mediante actividades o problemas de generalización de patrones (Warren y Cooper, 2006; Tanişli, 2011; Blanton et al., 2015; Mouhayar y Jourdak, 2015; Pinto, 2016) que favorecen el estudio de las regularidades y propiedades del patrón (Kieran, Pang, Schifter y Ng, 2016) y fomentan su capacidad de generalizar, representar, justificar y razonar con relaciones matemáticas (Blanton, Levi, Crites y Dougherty, 2011).

Estos problemas o actividades, como reconocen Benedicto, Jaime y Gutiérrez (2015, p. 154):

[...] presentan los primeros términos de una secuencia y piden calcular, por este orden, el término inmediato (el que sigue a los dados), un término próximo a los conocidos (de manera que se puedan utilizar tanto la representación geométrica como la aritmética para el cálculo de valores), un término lejano tal que, aunque esté asociado a un valor numérico, su representación gráfica sea costosa de llevar a cabo y sea más conveniente poner en marcha un proceso de generalización y, finalmente, formular una expresión (que se espera sea algebraica) para el término general de la secuencia.

$\mathrm{Al}$ involucrarse en la generalización de patrones, los estudiantes pueden reconocer un mismo patrón de manera diferente y usar una gran variedad de estrategias (El Mouhayar y Jurdak, 2015), en las que combinan enfoques visuales y numéricos (Rivera, 2010). Las estrategias usadas con más frecuencia, reportadas en la literatura, son:

- Contar a partir de un dibujo, que aparece en generalizaciones inmediatas y cercanas. Se basa en el conteo de los elementos de un término o etapa en un patrón figural (e. g. Stacey, 1989).

- La estrategia recursiva, que los estudiantes usan en generalizaciones cercanas para encontrar la diferencia común entre pares de términos consecutivos en un patrón y sumarla repetidamente de un término a otro para extender el patrón (e. g. Lannin, Barker y Townsend, 2006; Jurdak y El Mouhayar, 2014; El Mouhayar y Jurdak, 2015).

- Fragmentar, estrategia usada en generalizaciones cercanas y lejanas. Se basa en el patrón recursivo, al multiplicar la diferencia común entre pares de términos o etapas consecutivas en el patrón por el número de pasos y al sumar el resultado a un término figural inicial (e. g. Lannin et al., 2006; Jurdak y El Mouhayar, 2014; El Mouhayar y Jurdak, 2015).

- La estrategia funcional, que aparece en etapas lejanas, donde se relacionan las dos variables: la posición de la figura y el número de elementos de esta (Rivera, 2010; Jurdak y El Mouhayar, 2014), mediante una función afín, $f(n)=a n+b,(b \neq 0)$ que puede ser estrategia de función local si la relación se aplica a una determinada figura, o estrategias de función global, si la relación se aplica a una figura cualquiera (Zapatera, 2018). 
- Objeto-entero, que aparece con frecuencia en etapas lejanas. Implica identificar el valor de un término usando múltiplos de uno anterior o agregando dos términos anteriores (e. g. Stacey, 1989; Lannin et al., 2006; Jurdak y El Mouhayar, 2014).

Los estudios de estrategias de generalización de patrones lineales (e. g. Lannin et al., 2006; Rivera 2013; Zapatera, 2018; Jurdak y El Mouhayar, 2014) también han documentado los factores que parecen influir en el uso de las estrategias, destacan: $a$ ) la visualización (pobre o fuerte), $b$ ) la estructura matemática de la tarea, $c$ ) las estrategias previas, $d$ ) los valores de entrada (cercanos, múltiplos de valores conocidos previos, y valores lejanos) y e) las interacciones sociales con el maestro y otros estudiantes. Asimismo, la variedad de este tipo de factores puede conducir a cambios en el uso de estrategias en los estudiantes y progresar hacia otras más sofisticadas. La sofisticación de una estrategia la reconocen a partir de la flexibilidad o cambios que hacen en sus formas de proceder (Lannin et al., 2006). Del mismo modo, estas cambian durante un periodo de instrucción y son determinantes en la formación de generalizaciones constructivas y deconstructivas (Rivera y Becker, 2008), de la variación de su uso en todos los niveles ( $4 .^{\circ}$ a $11 .^{\circ}$ grado) (El Mouhayar y Jurdak, 2015). Sin embargo, falta identificar las formas de uso de las estrategias, no solo para reconocer dificultades de los niños en edades tempranas, sino también para explorar la naturaleza de estas y para nuestra creciente comprensión sobre el pensamiento algebraico en esta población y el diseño de tareas que los desafíen a usar estrategias más sofisticadas que contribuyan en la formulación de generalizaciones, con anterioridad a una instrucción sobre el uso de símbolos y notaciones algebraicas. Esta investigación, que avanza en esa dirección, se planteó las preguntas siguientes, que guiaron el estudio:

- ¿Qué estrategias utilizan los niños de quinto grado de primaria en la resolución de problemas de generalización de patrones?, y

- ¿cuáles son las diferentes formas de uso de cada tipo de estrategia?

\section{MARCO CONCEPTUAL}

\section{Patrón y generalización}

Varios autores han destacado la importancia de incorporar el estudio de patrones desde edades tempranas, lo que se justifica por que contribuye a que los estudiantes reconozcan regularidades, conecten relaciones y representen estas relaciones mediante símbolos (e. g., Radford, 2008). Un patrón, en el dominio de las matemáticas, en palabras de Mulligan y Milchelmore (2009, p. 34), es «cualquier regularidad que usualmente involucra relaciones numéricas, espaciales o lógicas». Además, es un paso fundamental en la formación de generalizaciones (Tanişli, 2011), por lo que los estudiantes deben tener experiencias previas con patrones para desarrollar su pensamiento algebraico mucho antes de instruirlos en el uso de la notación y simbología algebraica (Carraher y Schliemann, 2018).

La generalización «es un atributo común del pensamiento humano» (Radford, 2018, p. 7) y se refiere tanto al pensamiento individual como a la comunicación social (Dörfler, 1991). La generalización es definida por Kaput (1999, p. 136) como:

[...] extender deliberadamente el rango de razonamiento o comunicación más allá del caso o casos considerados, identificando explícitamente y exponiendo similitud entre casos, o aumentando el razonamiento o comunicación a un nivel donde el foco no son los casos o situación en sí mismos, sino los patrones, procedimientos, estructuras, y las relaciones a lo largo y entre ellos. 
Un aspecto esencial de la generalización descrita por Kaput es que, junto a la formalización, son intrínsecos a la actividad y al pensamiento matemático.

\section{Representación}

Las representaciones son parte esencial del proceso de aprendizaje de las matemáticas y conectan los objetos mentales con los objetos matemáticos (Rico, Castro y Romero, 2000). Se definen como: «aquellas herramientas -signos o gráficos- mediante las cuales los sujetos particulares abordan e interactúan con el conocimiento matemático. Mediante el trabajo con las representaciones las personas asignan significados a las estructuras matemáticas» (Rico, Castro y Romero, 2000, p. 1).

Las representaciones asociadas a los objetos matemáticos pueden organizarse en diferentes tipos de representación, de acuerdo con el énfasis en sus características y propiedades (Lupiáñez, 2016). En los patrones matemáticos se distinguen cuatro tipos de representación base: 1) figural, que usa únicamente recursos visuales, en general dibujos sin ningún tipo de notación simbólica (Merino, Cañadas y Molina, 2013); 2) numérica, que se sirve de números y operaciones expresadas mediante lenguaje matemático que suelen organizarse para realizar un cómputo; 3) verbal, que se distingue por el uso del lenguaje natural oral o escrito para exponer la información de forma cohesionada; y 4) algebraica, que se caracteriza por el uso del lenguaje algebraico para manifestar un enunciado o generalizar las operaciones aritméticas (Cañadas y Figueiras, 2011).

El trabajo con este tipo de representaciones contribuye a que los niños den significado a los objetos matemáticos representados, establezcan relaciones y comuniquen conceptos e ideas que generan al construir y expresar generalizaciones. Son un medio externo útil para que los niños interpreten y expliquen objetos matemáticos al resolver problemas de generalización de patrones.

\section{Estrategia}

Los problemas de patrones pueden ser resueltos mediante diversos procedimientos. Estos procedimientos suelen ser llamados estrategias, como en Rico (1997, p. 33), quien las define como «cualquier procedimiento o regla de acción que permite obtener una conclusión o responder a una cuestión haciendo uso de relaciones y conceptos, generales o específicos de una determinada estructura conceptual». En la resolución de problemas puede utilizarse más de una estrategia. Su uso, como se afirma en Rico (1997), supone un dominio de la red conceptual sobre la que deben ejercitarse y una dosis de creatividad e imaginación para descubrir nuevas relaciones o nuevos sentidos en relaciones ya conocidas.

Los tipos de estrategias de resolución de problemas de generalización de patrones, principalmente definidos y de interés para este estudio, son presentados y descritos en la sección de metodología.

\section{Formas de uso}

El concepto formas de uso se estableció en la literatura como una estrategia que puede presentar variaciones, reconocidas a través del razonamiento seguido y la forma de representar sus procedimientos. El primer fundamento lo establece Stacey (1989), al identificar en su estudio que los niños utilizaron ocasionalmente una variación del método de objeto-entero, usaron la suma $\mathrm{f}(12)+\mathrm{f}(8)$ para calcular $f(20)$ o ingeniosamente encontraron $f(1000)$ de $8 \times f(111)+f(112)$ en lugar de la multiplicación $f(20)=4 \times f(5)$ o $f(1000)=50 \times f(20)$.

Lannin et al. (2006) reconocen que una misma estrategia puede emerger de diferentes razonamientos. Por ejemplo, el uso de la estrategia recursiva puede aparecer por dos razones diferentes: 1) el niño 
podría determinar una regla recursiva basada en la comprensión de una relación que ocurre en un patrón figural; o 2) el niño podría descubrir un patrón numérico en los valores consecutivos de la variable dependiente, en ausencia de una conexión fuerte con el contexto figural de un patrón.

Lo anterior evidencia la importancia que tiene el especificar que, además de la diversidad entre estrategias, existen formas de ejecutar una misma estrategia. El presente estudio conceptualiza formas de uso de las estrategias a partir de los modos en los que los estudiantes ejecutan cada procedimiento o regla de acción para establecer una conclusión.

\section{METODOLOGÍA}

Se diseñó e implementó un experimento de enseñanza (Cobb, 2000), con el fin de desarrollar en los niños formas flexibles y productivas de pensamiento algebraico, en particular, las que refieren a la comprensión de relaciones funcionales lineales, que van desde relaciones específicas a generalizaciones de esas relaciones en todas sus instancias. En ese contexto, se les invitó a trabajar con seis problemas de generalización de patrones lineales en sus distintas representaciones, variedad en sus condiciones iniciales y relaciones funcionales afines de la forma general $f(n)=a n+b$, con $b \neq 0$.

\section{Experimento de enseñanza y entrevistas}

Se diseñaron seis problemas de generalización en un contexto de papel y lápiz, que desafiaron a los estudiantes a trabajar con patrones lineales de tipo figural, numérico y verbal. El experimento de enseñanza se desarrolló en seis sesiones de 120 minutos aproximadamente. Cada sesión abordó un problema y constó de cinco momentos: I) al inicio de la sesión, la profesora introdujo el problema mediante una lectura en voz alta; II) los niños trabajaron individualmente con el problema; III) durante el trabajo individual, fueron entrevistados sobre su forma de proceder al resolver el problema y se seleccionaron los niños con formas diferentes de proceder; IV) se motivó a estos niños a explicar a toda la clase, apoyados de la pizarra, la forma de proceder que siguieron para resolver el problema, y v) grupalmente estos niños respondieron preguntas y debatieron con sus compañeros sobre el proceso de solución al problema.

Las entrevistas individuales y las discusiones grupales contribuyeron a profundizar en las diferentes formas de proceder y en las decisiones que tomaron los niños al resolver los problemas.

El análisis de los resultados se centró en tres de los seis problemas de generalización (tabla 1), implementados en las sesiones tres, cinco y seis del experimento de enseñanza. El criterio para su elección atendió a que involucran representaciones de tipo figural, numérico y verbal. 
Tabla 1.

Problemas de generalización de patrones

\begin{tabular}{|c|c|c|c|}
\hline \multicolumn{3}{|c|}{ Problema } & Características \\
\hline \multicolumn{3}{|c|}{$\begin{array}{l}\text { P1: Las figuras de una sucesión están formadas por cuadrados blancos y grises } \\
\text { del mismo tamańo. Los cuadrados blancos se ubican de forma alineada, en el } \\
\text { centro de cada figura y los grises, alrededor de los blancos, como se muestra a } \\
\text { continuación. }\end{array}$} & $\begin{array}{l}\text { Sesión } 3 \\
\text { Relación funcional: } \\
f(n)=2 n+6 \text {. } \\
\text { Patrón figural tomado de Cañadas } \\
(2007, \text { p. } 209) \text {. }\end{array}$ \\
\hline \multicolumn{3}{|c|}{$\begin{array}{l}\text { P2: Analiza y completa la siguiente tabla que organiza los datos del número de } \\
\text { triángulos y la cantidad de palillos de una sucesión. }\end{array}$} & \multirow[t]{2}{*}{$\begin{array}{l}\text { Sesión } 5 \\
\text { Relación funcional: } \\
f(n)=2 n+1 \\
\text { Patrón adaptado de Radford (2008, } \\
\text { p. 86). }\end{array}$} \\
\hline \multicolumn{3}{|c|}{$\begin{array}{l}\text { a) Según los datos de la tabla, describe la relación que se establece entre el número } \\
\text { de triángulos y la cantidad de palillos. } \\
\text { b) Si el número de triángulos es } 55 \text { ¿Cuál es la cantidad de palillos que le corres- } \\
\text { ponde? ¿Y si el número de triángulos es } 231 \text { ? Argumenta tu respuesta. } \\
\text { c) Establece una regla para determinar rápidamente la cantidad de palillos que le } \\
\text { corresponde a cierto número de triángulos. Explica tu regla. }\end{array}$} & \\
\hline \multicolumn{3}{|c|}{$\begin{array}{l}\text { P3: Analiza la siguiente situación y responde lo que se te solicita. } \\
\qquad \begin{array}{l}\text { Un niño desea ahorrar de forma que cada día ahorre } \$ 3.00 \text {. } \\
\text { Inicialmente su alcancía tiene } \$ 10.00 \text {. }\end{array} \\
\text { a) ¿Cuánto dinero tendrá su alcancía al término de una semana de ahorrar? } \\
\text { Argumenta tu respuesta. } \\
\text { b) ¿Cuánto dinero tendrá su alcancía al cabo de } 30 \text { días de ahorrar? ¿Cuánto al } \\
\text { cabo de } 107 \text { días de ahorrar? Argumenta tu respuesta. } \\
\text { c) Establece una regla para determinar rápidamente la cantidad de dinero que } \\
\text { tendrá el niño en su alcancía al transcurrir cierta cantidad de días de ahorrar. } \\
\text { Explica tu regla. } \\
\text { d) Usa la regla para determinar rápidamente la cantidad de dinero que tendrá el } \\
\text { niño en su alcancía al cabo de un año de ahorrar. }\end{array}$} & $\begin{array}{l}\text { Sesión } 6 \\
\text { Relación funcional: } \\
f(n)=3 n+10 \text {. } \\
\text { Patrón propuesto por el presente } \\
\text { estudio. }\end{array}$ \\
\hline
\end{tabular}




\section{Participantes}

Participaron 25 niños (17 mujeres y 8 hombres) de quinto grado de una escuela primaria ubicada al suroeste de México, quienes en el momento del estudio no habían recibido ninguna preparación en la resolución de problemas de generalización de patrones. La elección tanto de la institución como de la población consideró las facilidades de acceso y las condiciones necesarias que el director de la escuela ofreció para la experimentación en el aula: espacios físicos, horarios de trabajo, grupo de estudiantes y la no intervención del profesor titular en el experimento de enseñanza.

El experimento de enseńanza fue guiado por la primera autora de este documento, quien asumió el rol de profesora-investigadora. Su función consistió en: 1) promover el desarrollo del pensamiento algebraico y la interacción en el aula respetando ideas y estableciendo cuestiones para enfocar a los estudiantes en los problemas, de manera individual y con toda la clase; 2) gestionar dudas, errores y dificultades presentes en los estudiantes; 3) desafiar a los estudiantes a verificar sus respuestas, y 4) realizar en tiempo real entrevistas individuales que fueron registradas en audio. Estas tres últimas funciones fueron apoyadas por tres investigadores, quienes, además, tomaron notas de campo, capturaron fotografías a la pizarra y grabaron en vídeo las discusiones grupales.

\section{Análisis de datos}

El análisis de las estrategias y sus formas de uso por los estudiantes al resolver los problemas consideraron las producciones escritas y verbales que presentaron en los momentos (ii) a $(v)$ durante el experimento de enseñanza. Asimismo, la clasificación de estrategias que la investigación ha documentado, en el marco de la resolución de problemas de patrones lineales. Se ejemplifica en el marco de P1.

Tabla 2.

Clasificación de estrategias en problemas de generalización de patrones

\begin{tabular}{|c|c|c|}
\hline Estrategia & Descripción & Ejemplo \\
\hline $\begin{array}{l}\text { Contar a partir de un } \\
\text { dibujo }\end{array}$ & $\begin{array}{l}\text { Cuentan los elementos de un término } \\
\text { figural particular en un patrón (Jurdak } \\
\text { y El Mouhayar, 2014). }\end{array}$ & $\begin{array}{l}\text { Hay } 10 \text { cuadrados grises para } 2 \text { cuadrados blancos. Al } \\
\text { contar los cuadrados que forman la figura. }\end{array}$ \\
\hline Recursiva & $\begin{array}{l}\text { Señalan el valor del cambio entre pares } \\
\text { de términos consecutivos y lo utilizan } \\
\text { para agregar repetidamente de un tér- } \\
\text { mino a otro para extender el patrón } \\
\text { (Jurdak y El Mouhayar, 2014). }\end{array}$ & $\begin{array}{l}\text { Hay } 14 \text { cuadrados grises para } 4 \text { cuadrados blancos. Al } \\
\text { conocer el número de cuadrados grises en la figura con } 3 \\
\text { cuadrados blancos y agregarle } 2 \text { (valor del cambio). Por } \\
\text { lo tanto, } 12+2=14 \text {. }\end{array}$ \\
\hline Diferencia & $\begin{array}{l}\text { Multiplican el valor de la posición por } \\
\text { el valor del cambio entre pares de tér- } \\
\text { minos consecutivos en el patrón (Sta- } \\
\text { cey, 1989). }\end{array}$ & $\begin{array}{l}\text { La figura con } 10 \text { cuadrados blancos tiene } 20 \text { cuadrados } \\
\text { grises. Al multiplicar los } 10 \text { cuadrados blancos por } 2 \text { (va- } \\
\text { lor del cambio), } 10 \times 2=20\end{array}$ \\
\hline $\begin{array}{l}\text { Objeto-entero } \\
\text { (en inglés whole- } \\
\text { object) }\end{array}$ & $\begin{array}{l}\text { Construyen el valor de un término } \\
\text { utilizando múltiplos de un término } \\
\text { anterior o agregando dos términos } \\
\text { anteriores (Stacey, 1989; Jurdak y El } \\
\text { Mouhayar, 2014). }\end{array}$ & $\begin{array}{l}\text { Si en la figura formada por } 10 \text { cuadrados blancos hay } \\
26 \text { cuadrados grises, entonces la cantidad de cuadrados } \\
\text { grises en la figura formada por } 50 \text { cuadrados blancos es } \\
26 \times 5=130 \text {. }\end{array}$ \\
\hline
\end{tabular}




\begin{tabular}{|l|l|l|}
\hline \multicolumn{1}{|c|}{ Estrategia } & \multicolumn{1}{|c|}{ Descripción } & \multicolumn{1}{c|}{ Ejemplo } \\
\hline $\begin{array}{l}\text { Fragmentar } \\
\text { (en inglés chunking) }\end{array}$ & $\begin{array}{l}\text { Basados en un patrón recursivo, cons- } \\
\text { truyen una unidad sobre valores cono- } \\
\text { cidos del atributo deseado (Lannin et } \\
\text { al., 2006). }\end{array}$ & $\begin{array}{l}\text { Para una figura con 10 cuadrados blancos hay 26 cua- } \\
\text { drados grises. Así que, para una figura con 50 cuadrados } \\
\text { blancos, tomaría 26 + 40(2) porque la cantidad de cua- } \\
\text { drados grises aumenta en 2 cada vez. }\end{array}$ \\
\hline $\begin{array}{l}\text { Felacionan partes del patrón con el } \\
\text { valor de posición de la figura (El Mou- } \\
\text { hayary Jurdak, 2015; Zapatera, 2018). }\end{array}$ & $\begin{array}{l}\text { En la figura con 50 cuadrados blancos, hay dos filas (su- } \\
\text { perior e inferior) de cuadrados grises con la misma can- } \\
\text { tidad de cuadrados blancos y seis cuadrados ubicados en } \\
\text { los costados. Por lo tanto, la cantidad de cuadrados grises } \\
\text { es (50 x 2) +6 }=106\end{array}$ \\
\hline Otras & $\begin{array}{l}\text { Refieren a las respuestas que usan estra- } \\
\text { tegias, acertadas o erróneas, distintas a } \\
\text { las anteriores o respuestas sin sentido } \\
\text { aparente (Zapatera, 2018). }\end{array}$ & $\begin{array}{l}\text { La figura formada por 10 cuadrados blancos tiene 26 } \\
\text { cuadrados grises. La figura, con 50 cuadrados blancos, } \\
\text { tiene 50 + 26 + 10 = 86 cuadrados grises. Suman valores } \\
\text { conocidos. }\end{array}$ \\
\hline
\end{tabular}

El análisis consideró cuatro fases.

- Fase 1. Se hizo una triangulación entre las producciones escritas, las transcripciones de las entrevistas y de las discusiones grupales. A partir de ello, se identificó y verificó el tipo de estrategias que usaron los niños y se categorizaron con base en la clasificación establecida (tabla 2). Para cada problema, se registraron en una tabla las estrategias identificadas por niño y por cuestión. Además, se realizaron anotaciones sobre sus formas de uso.

- Fase 2. Se registró en una tabla el número de niños que utilizó cada tipo de estrategia por problema y tipo de término demandado en las cuestiones planteadas. Además, se registró el número de nińos que dejaron en blanco o sin respuesta las cuestiones.

- Fase 3. Se identificaron las diferentes formas de uso de cada estrategia con base en el razonamiento que siguieron los nińos en la resolución de los problemas y la forma de representar sus procedimientos. Ello implicó agruparlos por tipo de estrategia, tomando como base las anotaciones hechas en la fase 1 y una nueva revisión de los datos para identificar similitudes y diferencias en sus modos de ejecución de cada procedimiento o regla de acción. En el proceso de caracterización se reconocieron algunos aspectos derivados de investigaciones previas, los cuales se contemplaron para establecer las diferentes categorías.

- Fase 4. Se reorganizó la tabla de la fase 2 en conexión con las formas de uso reconocidas por tipo de estrategia.

\section{RESULTADOS}

Desde los resultados del experimento de enseñanza, se reporta el análisis del tipo de estrategias y sus diferentes formas de uso que utilizan niños de quinto grado de primaria al resolver problemas de generalización de patrones lineales. Se estudiaron a partir de los razonamientos que siguieron y las formas de representar sus procedimientos.

\section{Tipos de estrategias y sus diferentes formas de uso}

A lo largo del experimento de enseñanza los niños siguieron diferentes formas de razonamiento y utilizaron formas diferentes de representar sus procedimientos. Se identificaron y caracterizaron siete tipos de estrategias y quince formas de su uso. Para cada tipo de estrategia se describen sus diferentes formas de uso con relación a un problema y un dato de ejemplo. Para respetar el anonimato de los participantes se utilizan seudónimos. 


\section{Recursiva}

Esta estrategia se evidenció en el proceso de resolución de los tres problemas y se articuló a los procedimientos que siguieron los niños para determinar términos inmediatos y cercanos. Su uso estuvo condicionado por el trabajo de patrones en su representación numérica, lo que implicó que transitaran de una representación figural (P1) o verbal (P3) a una numérica (simple o tabular) del patrón. En P2 la usó una mayoría (21 de 25 niños), cuyo patrón fue de tipo numérico-tabular.

El trabajo en esta estrategia lo ubicaron en el reconocimiento del cambio entre pares de términos consecutivos y lo usaron para extender el patrón. Su proceder consistió en agregar el valor del cambio repetidamente de un término a otro hasta el término deseado. Se identificaron tres formas diferentes de uso (tabla 4), que los llevó a determinar de forma correcta los términos demandados.

Tabla 4.

Formas de uso de la estrategia recursiva

\begin{tabular}{|c|c|}
\hline Forma de uso & Problema y datos de ejemplo \\
\hline $\begin{array}{l}\text { D. Suman repetidamente desde un término inicial. Parten } \\
\text { del término inicial y suman el valor del cambio de forma } \\
\text { iterada hasta el término requerido. }\end{array}$ & $\begin{array}{l}\text { P3: ¿Cuánto dinero tendrá su alcancía al término de una } \\
\text { semana de ahorrar? } \\
\text { Yahir [Producción escrita]: } \\
31 \text { pesos tendro su ul concia } \\
\text { por que se suman tres } \\
\text { pesos cado día, }\end{array}$ \\
\hline $\begin{array}{l}\text { E. Suman repetidamente desde un término cualquiera. Par- } \\
\text { ten de un término cualquiera y suman el valor del cambio } \\
\text { de forma iterada hasta el término requerido. }\end{array}$ & 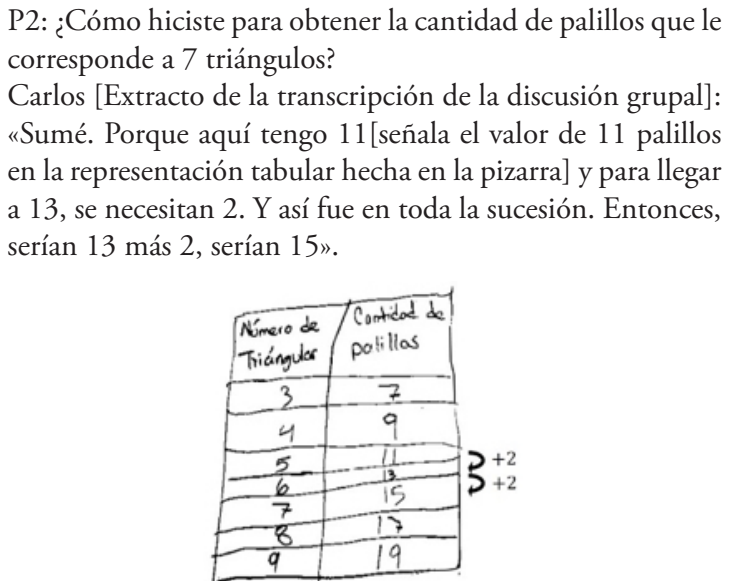 \\
\hline
\end{tabular}




\begin{tabular}{|c|c|c|c|c|}
\hline Forma de uso & \multicolumn{4}{|c|}{ Problema y datos de ejemplo } \\
\hline \multirow[t]{9}{*}{$\begin{array}{l}\text { F. Coordinan la suma repetida entre los aspectos cuantifica- } \\
\text { bles del patrón. Identifican y coordinan el cambio de las dos } \\
\text { variables (posición y término) para extender el patrón hasta } \\
\text { el término deseado. }\end{array}$} & \multicolumn{4}{|c|}{$\begin{array}{l}\text { P2: Analiza y completa la siguiente tabla que organiza los } \\
\text { datos del número de triángulos y la cantidad de palillos de } \\
\text { una sucesión. } \\
\text { Karen [Extracto de la transcripción de la entrevista indivi- } \\
\text { dual]: "Para completar la tabla, vi que el número de trián- } \\
\text { gulos se va sumando de uno en uno, tres más uno cuatro, } \\
\text { cuatro más uno cinco, y así se va, y aquí en la cantidad de } \\
\text { palillos se le va sumando de dos, porque siete más dos son } \\
\text { nueve, nueve más dos son once, y así se va sumando en esta } \\
\text { parte» [señala en la tabla la columna de valores de la canti- } \\
\text { dad de palillos]. }\end{array}$} \\
\hline & \multirow{8}{*}{${ }^{+1} \mathrm{C}$} & $\begin{array}{l}\text { Número de } \\
\text { triángulos }\end{array}$ & $\begin{array}{l}\text { Cantidad } \\
\text { de palillos }\end{array}$ & \multirow{8}{*}{$3_{+2}^{+2}$} \\
\hline & & 3 & 7 & \\
\hline & & 4 & 9 & \\
\hline & & 5 & 11 & \\
\hline & & 6 & 13 & \\
\hline & & 7 & 15 & \\
\hline & & 8 & 17 & \\
\hline & & 9 & 19 & \\
\hline
\end{tabular}

Las formas de uso D y E se asemejan, ya que parten de un término conocido, la variante ocurre en el término de su elección, el inicial u otro posterior. Resulta de descubrir un patrón de recursividad entre los primeros términos y continuarlo término a término para extender el patrón (D); o bien, de generalizar el patrón de recursividad para todos los términos y aplicarlo a uno posterior para extenderlo (E). Estas dos formas son reconocidas por Zapatera (2018) como subcategorías de la estrategia aditiva.

La forma de uso F se incluyó en esta estrategia, admitiendo la variación en su proceso de resolución de los niños de coordinar el patrón de recursividad de las dos variables del patrón. Proceso reconocido por Smith (2008, p. 147) como enfoque covariacional, al centrar la atención «en los cambios correspondientes en las variables individuales».

\section{Diferencia}

Esta regla de acción la empleó un niño en P1 y tres en P3, como estrategia posterior a la de contar y a la recursiva. Su uso conllevó asumir implícitamente que la suma repetida del valor del cambio implicaba $n \times a$, donde $n$ es la posición del término solicitado y a el valor del cambio. Su uso derivó de una interpretación inadecuada de los datos, al reconocerse lo que cambia en el patrón, sin percibir lo invariante, lo que originó respuestas erróneas a las cuestiones referidas a términos lejanos y el general. Se observó una sola forma de uso (tabla 5).

Tabla 5 .

Formas de uso de la estrategia de diferencia

\begin{tabular}{|c|c|}
\hline Forma de uso & Problema y datos de ejemplo \\
\hline $\begin{array}{l}\text { G. Multiplican el valor de la posición por el valor del } \\
\text { cambio. Asumen implícitamente que la suma iterada del } \\
\text { valor del cambio }(a) \text { implíca } f(n)=n \times a \text {, donde } n \\
\text { es el valor de la posición del término solicitado. }\end{array}$ & $\begin{array}{l}\text { P3: ¿Cuánto dinero tendrá en su alcancía al cabo de } 107 \text { días } \\
\text { de ahorrar? } \\
\text { Gladis [Producción escrita]: } \\
\qquad 107 \text { tendian } 107 \times 3=321 \\
107 \text { de los diaz } 4 \text { el } 3 \text { de lo ahotrado }\end{array}$ \\
\hline
\end{tabular}




\section{Objeto-entero}

Estrategia reconocida en el proceso de resolución de P1 y P2. Se articuló al proceder seguido por los nińos al determinar términos inmediatos, lejanos y el general. La antecedieron las estrategias de contar y recursiva. Se basaron en el análisis del patrón desde una representación numérica, donde establecieron relaciones e hicieron comparaciones entre los valores de las variables. En ese proceso, fijaron uno o dos términos conocidos para construir un término más grande o más pequeño, usando múltiplos, divisores o sumas de los términos conocidos. Se reconocieron dos diferentes formas de uso (tabla 6), derivadas de respuestas incorrectas a cuestiones sobre términos inmediatos, lejanos y el general.

Tabla 6.

Formas de uso de la estrategia objeto-entero

\begin{tabular}{|c|c|}
\hline Forma de uso & Problema y datos de ejemplo \\
\hline $\begin{array}{l}\text { H. Multiplican (o dividen) un término previo (o } \\
\text { posterior). Utilizan el múltiplo de un término pre- } \\
\text { vio para obtener el valor de un término más gran- } \\
\text { de }[f(m n)=m \times f(n)] \text { o usan el divisor de un } \\
\text { término posterior para determinar el valor de otro } \\
\text { más pequeño }\left[f\left(\frac{n}{m}\right)=\frac{f(n)}{m}\right] \text {. }\end{array}$ & $\begin{array}{l}\text { P1: ¿Cuántos cuadrados grises se colocarían en la figura, si se tienen } \\
\text { cuatro cuadrados blancos? } \\
\text { Dulce [Producción escrita]: } \\
16 \text { cuadros porque muitipique } 4 \times 4=16\end{array}$ \\
\hline $\begin{array}{l}\text { I. Suman dos términos previos. Emplean la } \\
\text { suma de dos términos previos para obtener el va- } \\
\text { lor de un término más grande. En ese proceso, } \\
\text { usan el múltiplo de uno de los términos previos } \\
{[f(m n+p)=m \times f(n)+f(p)] \text {. }}\end{array}$ & 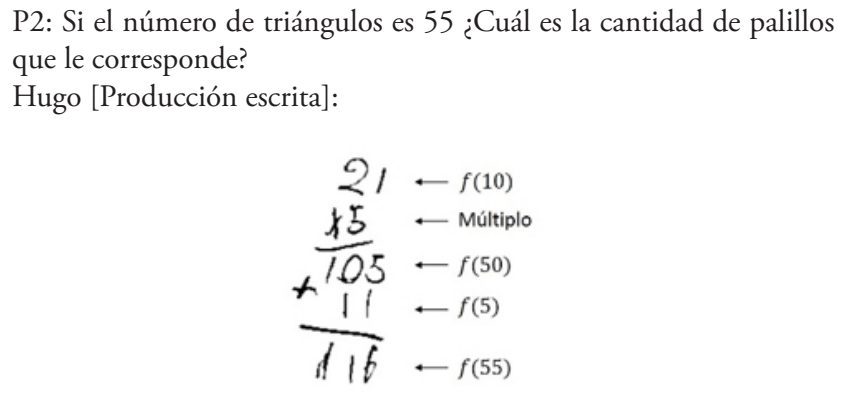 \\
\hline
\end{tabular}

Las diferencias entre las formas de uso H e I son la operación aritmética y la cantidad de términos usados para construir el término demandado, al reconocerlo como un múltiplo o divisor de un término conocido $(\mathrm{H})$ o como la suma de dos términos conocidos (I). Las primeras inferencias sobre estas dos formas de uso son destacadas por Stacey (1989).

\section{Fragmentar}

Esta estrategia la usaron dos niñas (una en P1 y otra en P3), al trabajar con términos cercanos y lejanos. Se usó como una regla de acción inmediata a la estrategia recursiva (P1) y como un procedimiento alterno a la estrategia funcional (P3). Su uso se sustentó en un patrón recursivo para construir una unidad sobre valores conocidos del patrón. Utilizaron los valores iniciales y el demandado. Se reconocieron las formas de uso J y K (tabla 7), que difieren en la coordinación (u omisión) del patrón de recursividad de las dos variables del patrón (posición y término) existentes entre el valor inicial y el demandado. La forma de uso J se caracterizó por considerar tal coordinación y por arribar a respuestas correctas de los términos demandados. La de tipo K, cuyas respuestas fueron erróneas, se distinguió por la ausencia de esa coordinación, al ser atendido solo el patrón de recursividad de la variable término. 
Tabla 7.

Formas de uso de la estrategia fragmentar

\begin{tabular}{|c|c|}
\hline Forma de uso & Problema y datos de ejemplo \\
\hline $\begin{array}{l}\text { J. Toman el valor del cambio y construyen una unidad } \\
\text { sobre valores conocidos. Toman el resultado de la dife- } \\
\text { rencia entre los valores de la posición deseada }(n) \text { con } \\
\text { el de una posición inicial conocida }(m) \text { y lo multiplican } \\
\text { por el valor del cambio }(a) \text {. Al final, le suman el valor del } \\
\text { término de la posición inicial conocida } f(m) \text { La regla de } \\
\text { acción es } f(n)=[(n-m) a]+f(m) \text {. }\end{array}$ & $\begin{array}{l}\text { P1: ¿Cuántos cuadrados grises se colocarían en la figura, si se } \\
\text { tienen } 50 \text { cuadrados blancos? } \\
\text { Fanny [Producción escrita]: } \\
\qquad \begin{array}{r}\text { para } 50: 106 \\
-\quad 3 \\
\frac{147}{94} \\
+\frac{12}{106}\end{array}\end{array}$ \\
\hline $\begin{array}{l}\text { K. Toman el valor del cambio y construyen una uni- } \\
\text { dad incorrecta sobre valores conocidos. Multiplican } \\
\text { el valor del cambio }(a) \text { por el valor de la posición de- } \\
\text { seada }(n) \text {. Al resultado, le adicionan el valor de un } \\
\text { término inicial conocido } f(m) \text {. La regla de acción es } \\
f(n)=(n \times a)+f(m) \text {. }\end{array}$ & $\begin{array}{l}\text { P3: ¿Cuánto dinero tendrá su alcancía al cabo de } 30 \text { días de } \\
\text { ahorrar? } \\
\text { Rosa [Producción escrita]: } \\
\qquad 50 \text { dias }=121 \\
\begin{array}{l}30 \\
\times 3 \\
+31\end{array}\end{array}$ \\
\hline
\end{tabular}

\section{Funcional}

Esta estrategia se usó en el proceso de resolución de los tres problemas y se articuló en procedimientos que siguieron los niños para encontrar términos cercanos, lejanos y el general. Su uso se vinculó a los cambios que hicieron en sus formas de proceder previas: contar, recursiva y diferencia. Se reconoce como la estrategia más utilizada e involucra la articulación de más de una relación entre los aspectos cuantificables del patrón (posición y término) en cada problema (tabla 8).

Tabla 8 .

Relaciones funcionales reconocidas en las resoluciones de los niños

\begin{tabular}{|c|c|c|}
\hline P1 & P2 & P3 \\
\hline$f(n)=(n+2)+(n+2)+2$ & $f(n)=n+n+1$ & $f(n)=\sum_{i=1}^{n} 3+10$ \\
$f(n)=n+n+6$ & $f(n)=3 n-(n-1)$ & $f(n)=3 n+10$ \\
$f(n)=2 n+6$ & $f(n)=2 n+1$ & \\
\hline
\end{tabular}

Basados en las relaciones establecidas, se identificaron tres formas de uso de esta estrategia. Para nombrar y describir estas categorías se consideró lo que la investigación ha documentado sobre el tipo de generalización que conlleva su estructura algebraica (constructiva o deconstructiva) y el tipo de expresión usado (estándar o no estándar). 
Una generalización es constructiva cuando se construye una expresión polinómica directa o cerrada, resultado de percibir cognitivamente las figuras o los valores numéricos del patrón como partes constituyentes no superpuestas. Es deconstructiva cuando se construye una expresión polinómica a partir de las etapas conocidas, como resultado de la percepción cognitiva de figuras o valores numéricos del patrón como partes o valores superpuestos (Rivera y Becker, 2008; Rivera, 2013).

Las generalizaciones constructivas y deconstructivas pueden expresarse de forma estándar y no estándar. Es estándar cuando los términos en la expresión directa correspondiente están en forma simplificada y no estándar cuando contiene términos que aún pueden simplificarse (Rivera, 2013).

La tabla 9 resume las tres diferentes formas de uso reconocidas en los procesos de resolución de los problemas.

Tabla 9.

Formas de uso de la estrategia funcional

\begin{tabular}{|c|c|}
\hline Forma de uso & Problema y datos de ejemplo \\
\hline $\begin{array}{l}\text { L. Establecen una generalización constructi- } \\
\text { va no estándar. Se basan en el patrón figural, } \\
\text { numérico o verbal y lo ven como compuesto } \\
\text { por partes o de valores numéricos no super- } \\
\text { puestos. La expresión matemática utilizada } \\
\text { está en su forma no simplificada. La relación } \\
\text { funcional del ejemplo es de la forma general } \\
f(n)=(n+2)+(n+2)+2 \text {. }\end{array}$ & 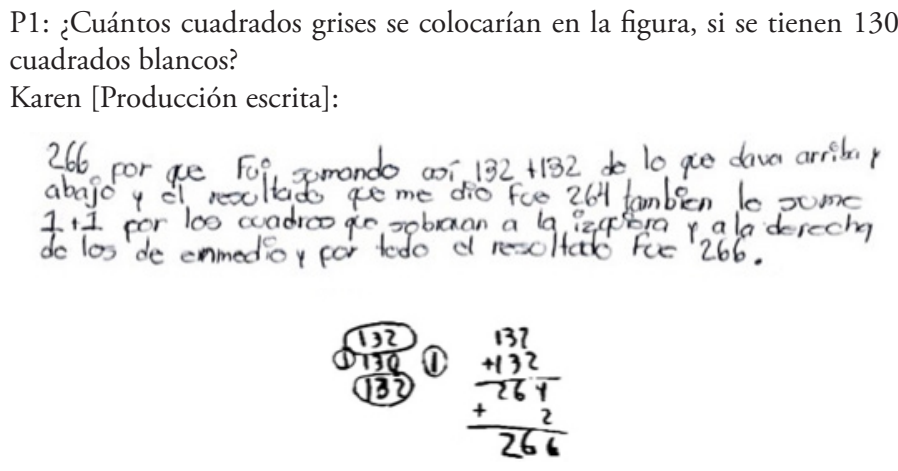 \\
\hline $\begin{array}{l}\text { M. Establecen una generalización decons- } \\
\text { tructiva no estándar. Se basan en el patrón } \\
\text { numérico, que, mediante un proceso de des- } \\
\text { composición, ven como compuesto de valo- } \\
\text { res superpuestos. La expresión matemática } \\
\text { utilizada está en su forma no simplificada. La } \\
\text { relación funcional del ejemplo tiene la forma } \\
\text { general } f(n)=3 n-(n-1) \text {. }\end{array}$ & $\begin{array}{l}\text { P2: Si el número de triángulos es } 231 \text { ¿Cuál es la cantidad de palillos que } \\
\text { le corresponde? } \\
\text { Gladis [Producción escrita]: } \\
\qquad \begin{array}{l}\frac{231}{693} \\
-\frac{230}{463}\end{array}\end{array}$ \\
\hline $\begin{array}{l}\text { N. Establecen una generalización constructi- } \\
\text { va estándar. Se basan en el patrón representa- } \\
\text { do de forma figural, numérico o verbal. Las } \\
\text { partes de la figura o los valores numéricos los } \\
\text { ven como compuestos de partes o valores no } \\
\text { superpuestos. La expresión matemática utili- } \\
\text { zada está en su forma simplificada. La rela- } \\
\text { ción funcional, del ejemplo, es de la forma } \\
\text { general } f(n)=3 n+10 \text {. }\end{array}$ & $\begin{array}{l}\text { P3: Establece una regla para determinar rápidamente la cantidad de dinero } \\
\text { que tendrá el niño en su alcancía al transcurrir cierta cantidad de días de } \\
\text { ahorrar. Explica tu regla. } \\
\text { Brenda [Producción escrita]: } \\
\text { Multiplicando los días por los } 3 \\
\text { pesos que el niño ahoria dionioy } \\
\text { sumarle los } 10 \text { que tienia al pirincipio. }\end{array}$ \\
\hline
\end{tabular}

\section{Otras}

Esta estrategia se identificó en el proceso de resolución de P3 y se articuló en el procedimiento seguido por uno de los niños para determinar términos lejanos y el general. Surgió como una manera alterna de 
proceder a la funcional. Su uso derivó de una inadecuada forma de relacionar los valores conocidos, al otorgarles un sentido aparente para obtener el término deseado, lo que conllevó respuestas incorrectas a las cuestiones articuladas a términos lejanos y el general (tabla 10).

Tabla 10.

Formas de uso de las otras estrategias

\begin{tabular}{|c|c|}
\hline Forma de uso & Problema y datos de ejemplo \\
\hline $\begin{array}{l}\text { O. Relacionan aparentemente valores conocidos. Parten } \\
\text { de valores conocidos y los relacionan otorgándoles un } \\
\text { sentido aparente para obtener el resultado del término } \\
\text { demandado. El procedimiento reconocido en P3 se resu- } \\
\text { me en } f(n)=[f(m) \times n]+10 \text {, donde } m \text { es el valor } \\
\text { de la posición de un término previo conocido }(f(m)), n \\
\text { el valor de la posición del término solicitado }(f(n)) \text { y } 10 \\
\text { la cantidad invariante en el problema. }\end{array}$ & $\begin{array}{l}\text { P3: Establece una regla para determinar rápidamente la canti- } \\
\text { dad de dinero que tendrá el niño en su alcancía al transcurrir } \\
\text { cierta cantidad de días de ahorrar. Explica tu regla. } \\
\text { Luis [Producción escrita]: } \\
\text { multiplicarlo por lo primero que ya } \\
\text { tenia ahorrado y por lo demas que } \\
\text { te piden. y poner los } 10 \$\end{array}$ \\
\hline
\end{tabular}

\section{DISCUSIÓN DE RESULTADOS}

Las estrategias y formas de uso se documentaron a partir de los razonamientos que siguieron los niños de quinto grado y las formas de representar sus procedimientos, en el proceso de resolución de problemas de generalización de patrones lineales. La tabla 11 resume estos resultados y muestra la cantidad de nińos que emplearon cada forma de uso (indicadas con letras del abecedario) en conexión con la estrategia de referencia y el tipo de término tratado en las cuestiones de cada problema. Además, reporta la cantidad de niños que no respondió a las cuestiones.

En P1, una niña evidenció dos formas de uso a la vez, J y N, asociadas a las estrategias de fragmentar y funcional respectivamente, para responder a las cuestiones que demandaban términos cercanos y lejanos. La figura 1 muestra la producción escrita de Fanny, se refiere a su forma de proceder para determinar términos del patrón. Primero utilizó una representación verbal escrita, para establecer una generalización constructiva estándar; la relación funcional asociada es $f(n)=2 n+6$. A continuación, manifestó una representación numérica que implicó la coordinación del patrón de recursividad de las dos variables del patrón (posición y término) para construir una unidad sobre valores conocidos (inicial y demandado). La regla de acción se resume como $f(n)=[(n-m) a]+f(m)$. La estrategia de fragmentación se asemeja a la funcional, aunque el valor inicial cambia a medida que intenta calcular nuevos valores del patrón o reafirmar su regla de acción. Por ejemplo, Fanny comenzó con el valor 3 para determinar el valor 50 (fig. 1, 1). Luego utilizó el valor 4 como el inicial para obtener el valor 50 (fig. 1,3); así confirmó que su procedimiento era válido independientemente del valor inicial ocupado. 


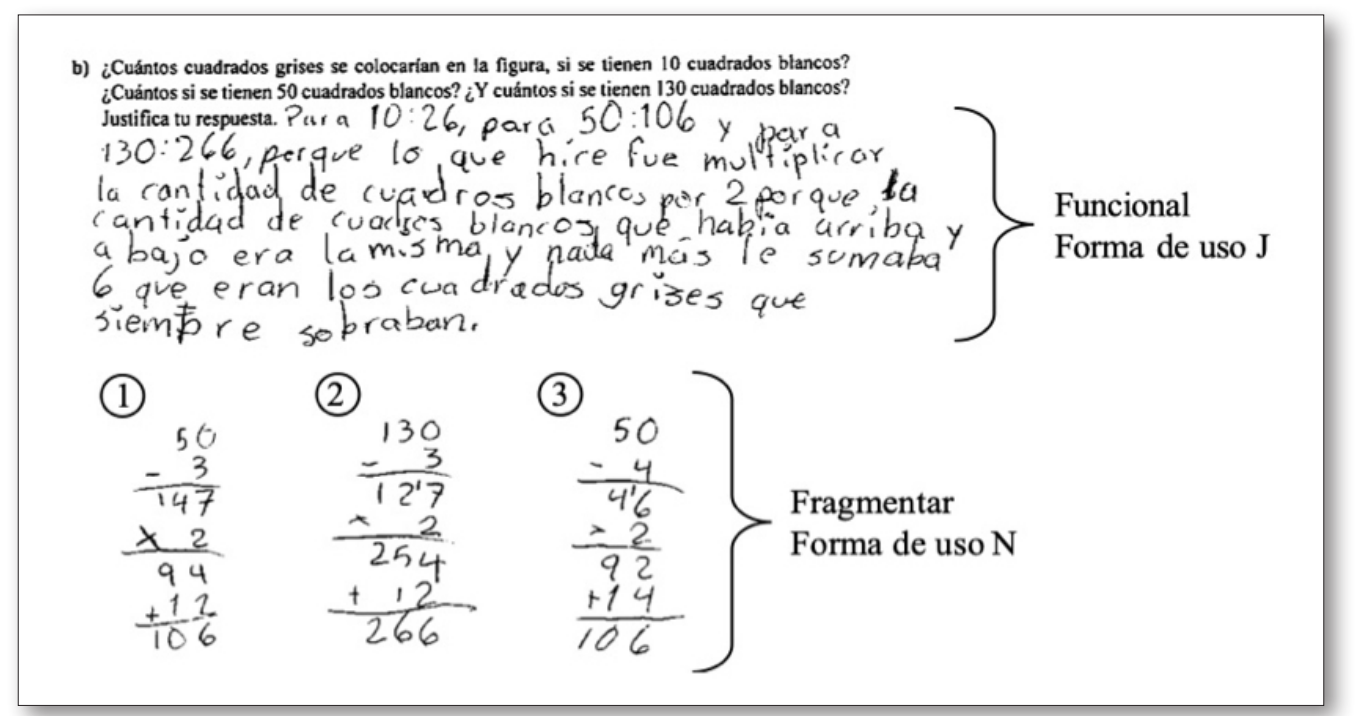

Fig. 1. Producción escrita de Fanny.

En ese contexto, la tabla 11 consideró dos veces a Fanny y, por ello, en algunos términos se presenta un total de 26 niños y en los demás de 25 niños (cantidad total de niños).

Tabla 11.

Estrategias y sus formas de uso utilizadas por los niños en el estudio

\begin{tabular}{|c|c|c|c|c|c|c|c|c|c|c|c|c|c|c|c|c|c|}
\hline \multirow{4}{*}{ Término } & \multicolumn{15}{|c|}{ Estrategia } & \multirow{4}{*}{ NR } & \multirow{4}{*}{$\begin{array}{c}\text { Total de } \\
\text { nińos }\end{array}$} \\
\hline & \multicolumn{3}{|c|}{ Contar } & \multicolumn{3}{|c|}{ Recursiva } & \multirow[t]{2}{*}{ Diferencia } & \multicolumn{2}{|c|}{$\begin{array}{c}\text { Objeto- } \\
\text { entero }\end{array}$} & \multicolumn{2}{|c|}{ Fragmentar } & \multicolumn{3}{|c|}{ Funcional } & Otras & & \\
\hline & \multicolumn{14}{|c|}{ Formas de uso } & & & \\
\hline & A & B & $\mathrm{C}$ & $\mathrm{D}$ & E & $\mathrm{F}$ & G & $\mathrm{H}$ & I & $\mathrm{J}$ & $\mathrm{K}$ & $\mathrm{L}$ & M & $\mathrm{N}$ & $\mathrm{O}$ & & \\
\hline \multicolumn{18}{|c|}{ P1 } \\
\hline Inmediato & 7 & 1 & 14 & 1 & - & - & - & 1 & - & - & - & - & - & 1 & - & - & 25 \\
\hline Cercano & 7 & - & - & - & - & - & - & - & - & 1 & - & 1 & - & 15 & - & 2 & 26 \\
\hline Lejano & - & - & - & - & - & - & 1 & 1 & - & 1 & - & 3 & - & 16 & - & 4 & 26 \\
\hline General & - & - & - & - & - & - & 1 & 1 & - & - & - & 2 & - & 14 & - & 7 & 25 \\
\hline \multicolumn{18}{|c|}{ P2 } \\
\hline Inmediato & - & - & - & 3 & 4 & 14 & - & - & - & - & - & - & 1 & 3 & - & - & 25 \\
\hline Lejano & - & - & - & - & - & - & 1 & - & 2 & - & - & 1 & 1 & 19 & - & 1 & 25 \\
\hline General & - & - & - & - & - & 1 & 1 & - & 1 & - & - & 1 & - & 17 & - & 4 & 25 \\
\hline \multicolumn{18}{|c|}{ P3 } \\
\hline Inmediato & - & - & - & 2 & - & - & - & - & - & - & - & 2 & - & 21 & - & - & 25 \\
\hline Lejano & - & - & - & - & - & - & 3 & - & - & - & 1 & - & - & 20 & 1 & - & 25 \\
\hline General & - & - & - & - & - & - & - & - & - & - & - & - & - & 23 & 1 & 1 & 25 \\
\hline
\end{tabular}

NR: No responde 


\section{Estrategias}

Según las estrategias utilizadas por niños de quinto grado de primaria, se reconoció que:

- Contar a partir de un dibujo fue usada solo en P1, en el contexto de un patrón figural. Bastó seguir la estructura espacial y numérica del patrón para determinar términos inmediatos y cercanos.

- La estrategia recursiva estuvo condicionada por el trabajo de patrones en su representación numérica. Ello implicó el tránsito en más de una representación para P1 y P3 y, así, se reconoció el valor del cambio entre pares de términos consecutivos y se utilizó para continuar el patrón en la determinación de términos inmediatos y cercanos.

- La estrategia de diferencia implicó el trabajo con representaciones numéricas de los tres patrones. Surge de una interpretación errónea de los datos, al identificar únicamente el cambio entre elementos sucesivos del patrón, sin percibir lo que no cambia. Se usó en términos lejanos y el general.

- Objeto-entero se evidenció en problemas que involucran representaciones figurales (P1) y numéricas (P2) del patrón. Su uso estuvo mediado por los valores de las posiciones de los términos conocidos y deseados, si eran múltiplos entre sí (por ejemplo, 10, 50, 100), factor identificado por Lannin et al. (2006) como potencialmente influyente en la selección de la estrategia objetoentero. A este tipo de términos cuyas posiciones consisten en múltiplos entre sí, Markworth (2010) los denominó términos seductores.

- La estrategia fragmentar se trabajó con representaciones numéricas en P1 y P3. Su uso se basó en un patrón recursivo para construir una unidad sobre valores conocidos del patrón, que se refieren al inicial y al demandado. Se usó para determinar términos cercanos y lejanos.

- La estrategia funcional se usó indistintamente de la representación del patrón. Los niños observaron la regla inmersa y la usaron prioritariamente para determinar términos cercanos, lejanos y el general. Quienes formularon la regla general lo hicieron de forma verbal para cualquier término o numérica asociada a un término lejano.

- En cuanto a otras estrategias, estas se observaron cuando transitaron de una representación verbal a una numérica en el patrón de P3. Se manifestó al establecer un procedimiento alterno al funcional para determinar términos lejanos y el general. En ese proceso, los nińos relacionaron valores de términos conocidos y la posición del término deseado, y les otorgaron un sentido «aparente», lo que condujo a una inadecuada relación de esos valores.

Esta variedad de estrategias evidencia cómo se involucran los niños para encontrar estrategias más eficaces a las utilizadas inicialmente en la resolución de los problemas, lo que supone modificar sus formas previas de proceder (e. g. contar y recursiva), y derivó en estrategias más sofisticadas (e. g. diferencia, objeto-entero, fragmentar, funcional y otras). La aplicación de estas últimas estrategias son intentos de los niños por calcular inmediatamente los términos demandados en las cuestiones de cada problema. En ese contexto, el uso de las estrategias de diferencia, objeto-entero y otras es resultado de una inadecuada interpretación de los datos del patrón, lo que derivó en respuestas incorrectas a las cuestiones planteadas. Por otro lado, la aplicación de las estrategias de contar, recursiva, fragmentar (en una de sus formas) y funcional es consecuencia de una adecuada interpretación del contexto del patrón, lo que favoreció llegar a resultados correctos. Certeza del desarrollo de formas flexibles y productivas de su pensamiento algebraico. 


\section{Formas de uso de las estrategias}

Los niños, al utilizar determinado tipo de estrategia, lo hacen con algunas variaciones. Surgen de diversas formas de razonar los problemas y de representar sus procedimientos. Esta vinculación entre estrategias y formas de uso (fig. 2) expresa la diversidad de caminos que siguen al resolver problemas de generalización de patrones. Dan pauta de cómo y hasta dónde son capaces de llegar en el desarrollo de su pensamiento algebraico y de la flexibilidad en su razonamiento (en el sentido de Krems, 1995) ante situaciones que desafían sus estructuras cognitivas.

Las formas de uso D, G, L y N fueron las únicas presentes y válidas en la resolución de los tres tipos de problemas (tabla 11). Las restantes parecieron estar condicionadas por la naturaleza de los problemas, pues su emergencia estuvo asociada a solo uno de estos.

La evidencia de tal condicionamiento se ve reflejada en dos formas de uso que la investigación ha destacado y que no fueron reconocidas en nuestros datos. La primera forma de uso, asociada a la estrategia recursiva, consiste en determinar una regla recursiva basada en la comprensión de una relación que ocurre en un patrón figural (Lannin et al., 2006). Su presencia está vinculada con el trabajo en problemas de generalización, donde el cambio entre pares de términos consecutivos es evidente en su configuración espacial del patrón figural. La segunda, asociada con la estrategia funcional, se refiere al establecimiento de una generalización deconstructiva estándar (Rivera, 2013). Su presencia suele darse cuando se trabaja con problemas con generalizaciones complicadas que implican una posible superposición de aspectos de las figuras del patrón (Rivera y Becker, 2008). Lo anterior enfatiza la necesidad de continuar profundizando en las formas de uso utilizando otro tipo de problemas a los establecidos.

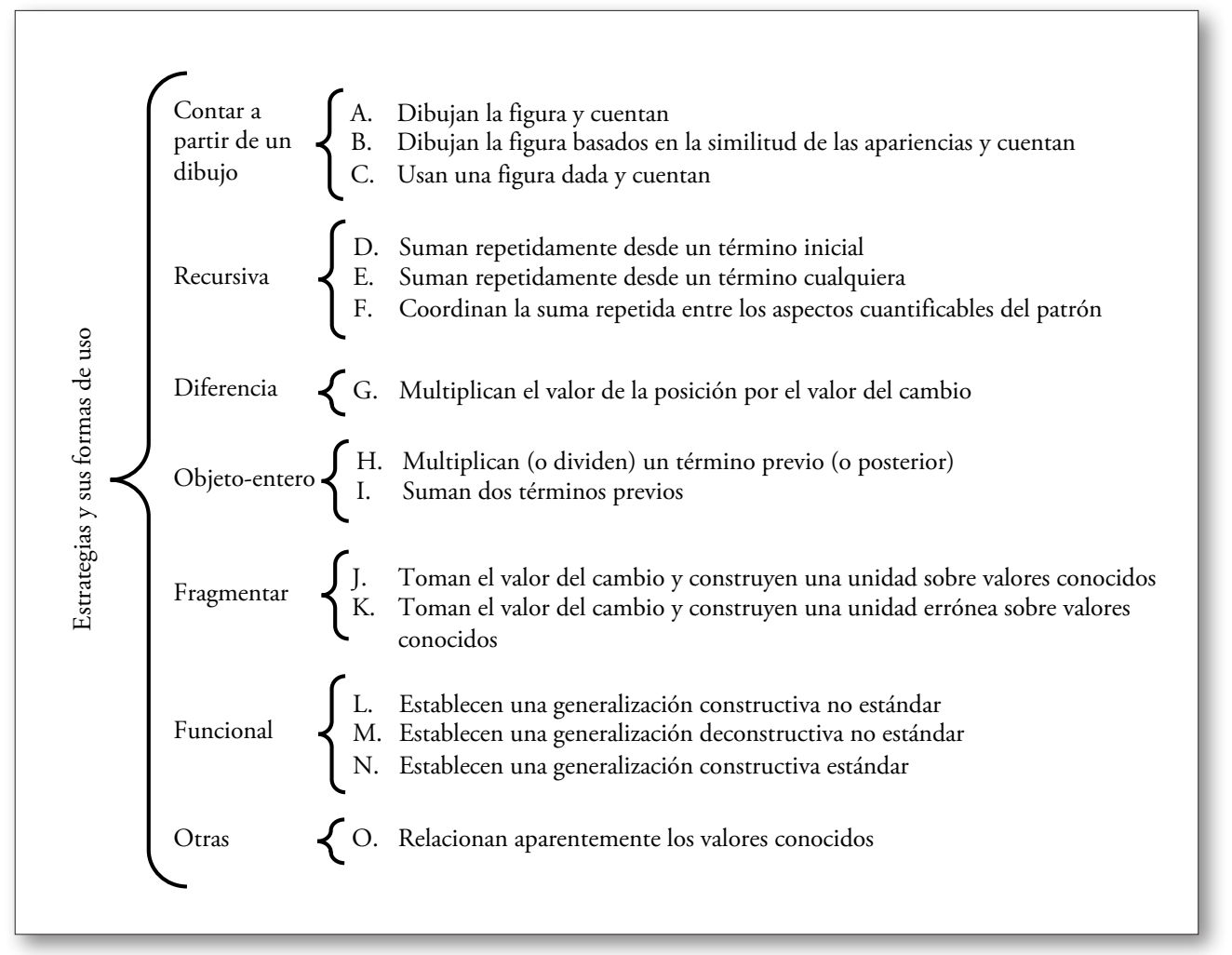

Fig. 2. Diferentes formas de uso de las estrategias. 


\section{CONCLUSIONES}

Este artículo indagó las estrategias empleadas por niños de quinto grado y caracterizó sus formas de uso (fig. 2) en la resolución de problemas de generalización de patrones lineales de tipo figural, numérico y verbal durante un experimento de enseñanza. Hay certeza de que las estrategias y sus formas de uso estuvieron condicionadas por: 1) la naturaleza de los problemas trabajados (tipo de patrón lineal involucrado, términos iniciales dados y el valor del término demandado en las cuestiones); 2) el trabajo con ciertas formas de representar los patrones; y 3) el razonamiento seguido por los niños al resolver los problemas.

Los resultados contribuyen a la investigación sobre el desarrollo del pensamiento algebraico. Informan sobre los diferentes modos de trabajar cada una de las estrategias, Estos han sido destacados de manera aislada para algunas estrategias (e. g. Stacey, 1989; Lannin et al., 2006; Rivera, 2013; Zapatera, 2018). Este artículo da visibilidad a este constructo y aporta una primera clasificación, conocimiento importante para investigadores y profesores, al ofrecer una ventana de oportunidades para el diseño y la gestión en el aula de clase de matemáticas de problemas que desafíen a los niños a usar no solo más de una estrategia y una forma de uso, sino estrategias y formas de uso más sofisticadas que contribuyan a la formulación de generalizaciones.

Se sugiere continuar con estudios que: 1) consideren niños de todos los grados de educación primaria y problemas con condiciones diferentes a los presentados; 2) analicen la influencia del trabajo en grupo en la estrategia y su forma de uso de un niño y sus implicaciones; y 3) examinen el comportamiento de un niño a lo largo de un experimento de enseñanza y su coherencia con la forma de uso cuando trabaja con la misma estrategia en problemas diferentes. A fin de obtener mayores explicaciones sobre la clasificación obtenida.

\section{REFERENCIAS}

Benedicto, C., Jaime, A. y Gutiérrez, Á. (2015). Análisis de la demanda cognitiva de problemas de patrones geométricos. En C. Fernández, M. Molina y N. Planas (Eds.), Investigación en Educación Matemática XIX (pp. 153-162). Alicante: SEIEM.

Blanton, M., Levi, L., Crites, T. y Dougherty, B. (2011). Developing essential understanding of algebraic thinking for teaching mathematics in grades 3-5. Reston, VA: National Council of Teachers of Mathematics.

Blanton, M., Stephens, A., Knuth, E., Gardiner, A., Isler, I. y Kim, J. (2015). The development of children's algebraic thinking: The impact of a comprehensive early algebra intervention in third grade. Journal for Research in Mathematics Education, 46(1), 39-87.

https://doi.org/10.5951/jresematheduc.46.1.0039

Blanton, M. L. y Kaput, J. J. (2005). Helping elementary teachers build mathematical generality into curriculum and instruction. $Z D M, 37(1), 34-42$.

https://doi.org/10.1007/bf02655895

Cañadas, M. C. (2007). Descripción y caracterización del razonamiento inductivo utilizado por estudiantes de educación secundaria al resolver tareas relacionadas con sucesiones lineales y cuadráticas (tesis doctoral no publicada). Universidad de Granada, España. http://funes.uniandes.edu.co/282/

Cañadas, M. C. y Figueiras, L. (2011). Uso de representaciones y generalización de la regla del producto. Infancia y Aprendizaje, 34(4), 409-425.

https://doi.org/10.1174/021037011797898449 
Carraher, D. W. y Schliemann, A. D. (2018). Cultivating early algebraic thinking. En C. Kieran (Ed.), Teaching and learning algebraic thinking with 5- to 12-year-olds: The global evolution of an emerging field of research and practice. ICME-13 Monographs (pp. 107-138). Chaim, Suiza: Springer. https://doi.org/10.1007/978-3-319-68351-5_5

Cobb, P. (2000). Conducting teaching experiments in collaboration with teachers. En A. E. Kelly y R. A. Lesh (Eds.), Handbook of research design in mathematics and science education (pp. 307-334). Mahwah, NJ: Lawrence Erlbaum Associates.

Dörfler, W. (1991). Forms and means of generalization in mathematics. En A. J. Bishop, S. MellinOlson y J. Van Dormolen (Eds.), Mathematical Knowledge: Its growth through teaching (pp. 63-88). Dordrecht: Springer. https://doi.org/10.1007/978-94-017-2195-0_4

El Mouhayar R. y Jurdak, J. (2015). Variation in strategy use across grade level by pattern generalization types. International Journal of Mathematical Education in Science and Technology, 46(4), 553-569. https://doi.org/10.1080/0020739x.2014.985272

Jurdak, M. E. y El Mouhayar, R. R. (2014). Trends in the development of student level of reasoning in pattern generalization tasks across grade level. Educational Studies in Mathematics, 85(1), 75-92. https://doi.org/10.1007/s10649-013-9494-2

Kaput, J. (1999). Teaching and learning a new algebra. En E. Fennema y T. A. Romberg (Eds.), Mathematics classrooms that promote understanding (pp. 133-155). Mahwah, NJ: Lawrence Erlbaum Associates.

Kieran, C., Pang, J., Schifter, D. y Ng, S. F. (2016). Early algebra. Research into its nature, its learning, its teaching. Nueva York: Springer.

https://doi.org/10.1007/978-3-319-32258-2

Krems, J. F. (1995). Cognitive flexibility and complex problem solving. En P. A. Frensch y J. Funke (Eds.), Complex problem solving: The European Perspective (pp. 201-218). Nueva York: Psychology Press.

Lannin, J., Barker, D. y Townsend, B. (2006). Algebraic generalisation strategies: factors influencing student strategy selection. Mathematics Education Research Journal, 18(3), 3-28. https://doi.org/10.1007/BF03217440

Lupiáñez, J. (2016). Sistemas de representación. En L. Rico y A. Moreno (Eds.), Elementos de didáctica de la matemática para el profesor de secundaria (pp. 119-137). Granada, España: Ediciones Pirámide.

Markworth, K. A. (2010). Growing and growing: promoting functional thinking with geometric growing patterns (tesis doctoral no publicada). University of North Carolina, Chapel Hill. https://core. ac.uk/download/pdf/210600357.pdf

Merino, E., Cañadas, M. C. y Molina, M. (2013). Uso de representaciones y patrones por alumnos de quinto de educación primaria en una tarea de generalización. Edma 0-6: Educación Matemática en la Infancia, 2(1), 24-40.

Mulligan, J. y Mitchelmore, M. (2009). Awareness of pattern and structure in early mathematical development. Mathematics Education Research Journal, 21(2), 33-49.

https://doi.org/10.1007/BF03217544

Pinto, E. (2019). Relaciones funcionales, sistemas de representación y generalización en estudiantes de tercero de primaria (trabajo final de máster no publicado). Universidad de Granada, España.

Radford, L. (2018). The Emergence of Symbolic Algebraic Thinking in Primary School. En C. Kieran (Ed.), Teaching and learning algebraic thinking with 5- to 12-year-olds: The global evolution of an emerging field of research and practice. ICME-13 Monographs (pp. 3-25). Nueva York: Springer. https://doi.org/10.1007/978-3-319-68351-5_1 
Radford, L. (2008). Iconicity and contraction: a semiotic investigation of forms of algebraic generalizations of patterns in different contexts. $Z D M, 40(1), 83-96$.

https://doi.org/10.1007/s11858-007-0061-0

Rico, L. (1997). Consideraciones sobre el currículo de matemáticas para educación secundaria. En L. Rico, E. Castro, E. Castro, M. Coriat, A. Marín, L. Puig, M. Sierra y M. M. Socas (Eds.), La educación matemática en la enseñanza secundaria (pp. 15-38). Madrid: ice-Horsori.

Rico, L., Castro, E. y Romero, I. (2000). Sistemas de representación y aprendizajes de estructuras numéricas. Granada, España: Universidad de Granada.

Rivera, F. (2013). Teaching and learning patterns in school mathematics. Psychological and pedagogical considerations. Nueva York: Springer.

https://doi.org/10.1007/978-94-007-2712-0

Rivera, F. D. (2010). Visual templates in pattern generalization activity. Educational Studies in Mathematics, 73(2), 297-328.

https://doi.org/10.1007/s10649-009-9222-0

Rivera, F. D. (2018). Pattern generalization processing of elementary students: cognitive factors affecting the development of exact mathematical structures. Eurasia Journal of Mathematics, Science and Technology Education, 14(9), em1586.

https://doi.org/10.29333/ejmste/92554

Rivera, F. D. y Becker, J. R. (2008). Middle school children's cognitive perceptions of constructive and deconstructive generalizations involving linear figural patterns. $Z D M, 40(1), 65-82$. https://doi.org/10.1007/s11858-007-0062-z

Smith, E. (2008). Representational thinking as a framework for introducing functions in the elementary curriculum. En J. J. Kaput, D. W. Carraher y M. L. Blanton (Eds.), Algebra in the early grades (pp. 133-160). Nueva York: Routledge.

Stacey, K. (1989). Finding and using patterns in linear generalising problems. Educational Studies in Mathematics, 20(2), 147-164.

https://doi.org/10.1007/BF00579460

Warren, E. y Cooper, T. (2006). Using repeating patterns to explore functional thinking. Australian Primary Mathematics Classroom, 11(1), 9-14.

Zapatera, A. (2018). Cómo alumnos de educación primaria resuelven problemas de generalización de patrones. Una trayectoria de aprendizaje. Revista Latinoamericana de Investigación en Matemática Educativa, 21(1), 87-114.

https://dx.doi.org/10.12802/relime.18.2114 


\title{
Pattern generalization strategies and their different forms of use in fifth grade
}

\author{
Melby Cetina-Vázquez \\ Centro de Investigación en Matemática Educativa, Universidad Autónoma de Guerrero, México \\ mgcetinav@uagro.mx \\ Guadalupe Cabañas-Sánchez \\ Centro de Investigación en Matemática Educativa, Universidad Autónoma de Guerrero, México \\ gcabanas@uagro.mx
}

The article reports the strategies used by elementary school children, along with the different forms of use in solving three linear pattern generalization problems of figural, numerical and verbal types. The study is based on the proceeding forms and the different representational forms used to stablish a conclusion or answer a question, during a teaching experiment. The participants were 25 fifth-grade children from an elementary school located in southwestern Mexico, who had not received any explicit training on solving generalization problems. The selection both the institution and the population, considered the facilities and the necessary conditions that the school principal provided for experimentation in the classroom.

In this study, strategy is understood as «Any procedure or rule of action that allows obtaining a conclusion or answering a question making use of relations and concepts, general or specific, of a certain conceptual structure» (Rico, 1997, p. 33). On the other hand, the concept of forms of use was established to recognize in the literature that a strategy can present variations, recognized through the reasoning followed and the way of representing its procedures.

Data was collected through six generalization problems during six sessions in a teaching experiment. Each sessions consisted of five moments: (i) the teacher introduced the problem by reading aloud; (ii) children worked individually on the problem; (iii) during individual work, they were interviewed about their proceeding forms when solving the problem and the children with different proceeding forms were selected; (iv) these children were motivated to explain to the whole class, supported by the blackboard, the proceeding forms they followed to solve the problem; $(v)$ at the whole class level these children answered questions and debated with their classmates about the process of solving the problem. The learning goal was to develop flexible and productive forms of algebraic thinking in children, particularly, those that refer to the understanding of linear functional relationships, ranging from specific relationships to generalizations of those relationships in all their instances.

Data analysis considered the following phases: Phase 1 . To triangulate the written productions, the transcripts of interviews and the group discussions in order to verify and categorize the type of strategies used by the children. Phase 2 . To record on a table the number of children who used each type of strategy by problem and type of demanded term in the proposed questions. Phase 3. To identify the different forms of use of each strategy based on the reasoning followed by the children in solving the problems and the form to represent their procedures. Phase 4. To reorganize the table of phase 2 in connection with the forms of use recognized by type of strategy.

Data analysis reveals seven types of strategies and fifteen forms of use in fifth grade primary school children. It is highlighted that counting and recursive strategies contributed in the children to the use of more sophisticated strategies such as: difference, whole-object, chunking, functional and others. These strategies presented one of three different forms of use. This link between strategies and forms of use expresses the diversity of paths they follow when solving pattern generalization problems. They give an indication of how and how far they are able to go in the development of their algebraic thinking and flexibility in their reasoning (in the sense of Krems, 1995) in situations that challenge their cognitive structures. It is certain that the strategies and their forms of use were conditioned by: 1) the nature of the worked problems (type of linear pattern involved, initial terms given and the value of the demanded term in the questions); 2) working with certain forms of representing patterns; and 3) the reasoning followed by children when solving problems. 